Journal of Computer Science 8 (4): 449-453, 2012

ISSN 1549-3636

(C) 2012 Science Publications

\title{
Multiple Input Multiple Output System with Space Time Block Coding and Orthogonal Frequency Division Multiplexing
}

\author{
${ }^{1}$ Ramesh Kumar, P. and ${ }^{2}$ R.M.S. Parvathi \\ ${ }^{1}$ Anna University of Technology, Coimbatore, Tamil Nadu, India \\ ${ }^{2}$ Senguthar College of Engineering, \\ Anna University of Technology, Tamil Nadu, India
}

\begin{abstract}
Problem statement: A large amount of research has been performed to achieve very high data rate networks to support reliable transmission of video, data and speech at high rates to many users. One way to increase the data rate in a wireless system is to use multiple transmit and/or receive antennas (MIMO structure). Indeed, it has been shown that the Shannon capacity of MIMO channel is (if the channel is known to the receiver) grows linearly with the number of transmit antennas and the number of receive antennas. MIMO systems can be implemented using various types of pre coding and modulation technique. All leads to get better signal at the receiver. Approach: Large scale fading due to multipath propagation of wireless signals can be mitigated by deploying multiple antennas both at the transmitter and the receiver. MIMO systems can be implemented using many techniques. Here at the transmitter the signals are coded using space time block codes and then they are modulated using Orthogonal Frequency Division Multiplexing (OFDM). Due to this the received signal can have an improved SNR. Results: The results are obtained for simple STBC-MIMO system, when it implemented for various number pf transmit and receive antennas. The resultant SNR and BER were obtained through simulation. Then the same system was deployed using OFDM technique. The results were compared for simple MIMO-OFDM and coded MIMO-OFDM. Conclusion/Recommendations: The complexity increases for higher data rate because of using both coding and modulation technique. By using proper mapping technique before deployment on the transmit antennas the complexity may be reduced. The OFDM technique can be improved to give high SNR by properly coding it like a CDMA process.
\end{abstract}

Key words: Multiple Input Multiple Output (MIMO), space time coding, multipath fading, SingleInput Single-Output (SISO), Orthogonal Frequency Division Multiplexing (OFDM)

\section{INTRODUCTION}

Wireless is the fastest growing segment of the communications market in the world. It has a wide range of services from satellites that provide low bit rates but global coverage and cellular systems with continental coverage to high bit rate local area networks and personal area networks with a maximum range of a few to a hundred meters. Using a cellular system is by far the most common wireless method to access data or perform voice dialing. In the near future, we will expect seamless global roaming across different wireless networks and ubiquitous access to personalized applications and rich content via a universal and userfriendly interface. Yet, in this climate, researchers still struggle with the fundamental questions about the physical limitations of communicating over wireless channel. These include multipath fading, limited spectrum resources, multiple-access interference and limited battery life of mobile devices (Alamouti, 1998; Abreu and Kohno, 2003; Dumard and Zemen, 2007; Hammons and Gamal, 2000; Kaiser, 2005; Liu et al., 2002; Tarokh et al., 1998; 1999; Kwon et al., 2010; Lan, 2010).

Consider the use of multiple antenna elements at both the transmitter and the receiver ends to improve a wireless connection. The use of multiple antennas has been a recent significant breakthrough in wireless technologies. It creates a Multi-Input Multi-Output (MIMO) channel in which each path from one transmit antenna to one receive antenna can be viewed as one signaling branch. MIMO systems have two major attractive advantages that conventional Single-Input Single-Output (SISO) systems do not have. These are:

Multiplexing gain (or spectral efficiency gain): As supported by information-theoretic studies the channel

Corresponding Author: Ramesh Kumar, P., Anna University of Technology, Coimbatore, Tamil Nadu, India 
capacity of a multiple-antenna system is considerably higher than that of a single-antenna system. In particular, it is widely understood that channel capacity increases asymptotically linearly with the minimum number of transmit and receive antennas when channel knowledge is available at the receiver. Therefore, the degree of freedom for communications is increased. As a result, the transmission rate increases linearly without an increase in the total transmission power or channel bandwidth.

Diversity gain: If the antennas at both ends have no, or very low, correlation, the signaling branches between different transmit-receive antenna pairs in a MIMO system can be assumed to be statistically independent. These independent branches create diversity gain. By transmitting the same data (in the same, or different, representations) over multiple independent branches, fading can be effectively mitigated and hence, link reliability significantly improved.

MIMO systems also provide other types of gains such as array gain and interference suppression gain. Consequently, multiple antennas are expected to play an important role in advanced wireless systems, for example, $3 \mathrm{G}$ and beyond.

The problem discussed in our research is how to develop fundamental transmission strategies adapted to a point-to-point wireless link with flat fading channels to utilize the promises of multiple antennas jointly or individually. This topic has, in fact, received much attention in the past few years. As the core idea is complementing the traditional time dimension with the space dimension inherently brought by multiple antennas, MIMO-related transmission strategies are often referred to as Space-Time (ST) techniques.

There is ST coding and modulation schemes that do not require channel knowledge, i.e., Channel State Information (CSI), at the transmitter. Both cases in which CSI is available (coherent) and unavailable (noncoherent) at the receiver, respectively, are considered

\section{Fundamentals of MIMO systems:}

Basic system design: Consider the system shown in Fig. 1 which employs multiple antennas on both the transmitter and the receiver side. Raw bit stream of digital data enters into the transmitter where it is passed through a simplified block that includes functions like coding which adds redundancy to the binary data in order to combat the detrimental effects of the channel, modulation which maps the binary stream into complex modulation symbols and mapping which includes assigning weights to different transmit antennas or spatial processing and beam forming or preceding. This signal is then up-converted to some higher frequency and sent through the Tx antennas simultaneously into the wireless channel. The signal which is received might be a different version of the transmitted signal as a result of the multi path fading and interferences undergone by the signal while passing through the channel. Hence the function of the receiver is to first demap, demodulate and decode the received signal and then form a decision of what signal was transmitted.

At the receiver:

$\mathrm{r}=\mathrm{Hs}+\mathrm{n}$

r- (nR x 1), s-(nT x 1), n-(nR x 1) iid h $0, \mathrm{~N})$

$\mathrm{H}$, (nR x nT) memory less, complex channel matrix

Assume the receiver have the appropriate knowledge of the channel known as Channel State Information (CSI). This can be accomplished by sending pilot waves on time to time basis. The bit sequence transmitted can be considered as:

$\mathrm{x}^{(\mathrm{i})}=\left[\mathrm{x}_{0}^{(\mathrm{i})}, \mathrm{x}_{1}^{(\mathrm{i})}, \ldots, \mathrm{x}_{\mathrm{M}-1}^{(\mathrm{i})}\right]$

This can be processed and received with channel knowledge by using the expressions:

$$
\begin{aligned}
& \tilde{\mathrm{X}}^{(\mathrm{i})}=[\underbrace{\mathrm{x}_{0}^{(\mathrm{i})}, \mathrm{x}_{1}^{(\mathrm{i})}, \ldots, \mathrm{x}_{\mathrm{M}-1}^{(\mathrm{i})}}_{\mathrm{K} \text { time }}]=\left[\tilde{\mathrm{x}}_{0}^{(\mathrm{i})}, \tilde{\mathrm{x}}_{1}^{(\mathrm{i})}, \ldots, \tilde{\mathrm{x}}_{\mathrm{N}-1}^{(\mathrm{i})}\right] \\
& \phi_{\mathrm{q}}^{(\mathrm{i})}=\mathrm{q} \frac{2 \pi}{\mathrm{N}},(\mathrm{q}=0 \ldots \mathrm{N}-1) \\
& \mathrm{y}^{(\mathrm{i})}=\left[\tilde{\mathrm{x}}_{0}^{(\mathrm{i})}, \tilde{\mathrm{x}}_{1}^{(\mathrm{i})} \mathrm{e}^{-\mathrm{j} \phi_{q}^{(\mathrm{i})}}, \ldots, \tilde{\mathrm{x}}_{2}^{(\mathrm{i})} \mathrm{e}^{-\mathrm{j} 2 \phi_{q}^{(\mathrm{i})}} \ldots ., \tilde{\mathrm{x}}_{\mathrm{N}-1}^{(\mathrm{i})} \mathrm{e}^{-\mathrm{j}(\mathrm{N}-1) \phi_{q}^{(\mathrm{i})}}\right]
\end{aligned}
$$

OFDM basics: The block diagram of OFDM system studied in this study is shown in Fig. 2. A serial-toparallel buffer segments the information bit sequence into parallel output stream and then modulator blocks map them into complex numbers which determines the constellation points of each sub carrier. The number of bits assigned to each sub carrier is variable based on the variability of signal to noise ratio across the frequency range. Optimization of this bit assignment will be detailed in further.

The OFDM modulation can be efficiently implemented in discrete time using an inverse FFT (IFFT) to act as a modulator and an FFT to act as a demodulator.

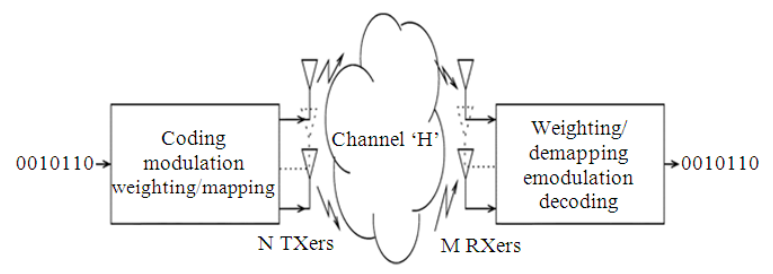

Fig. 1: MIMO wireless transmission system 


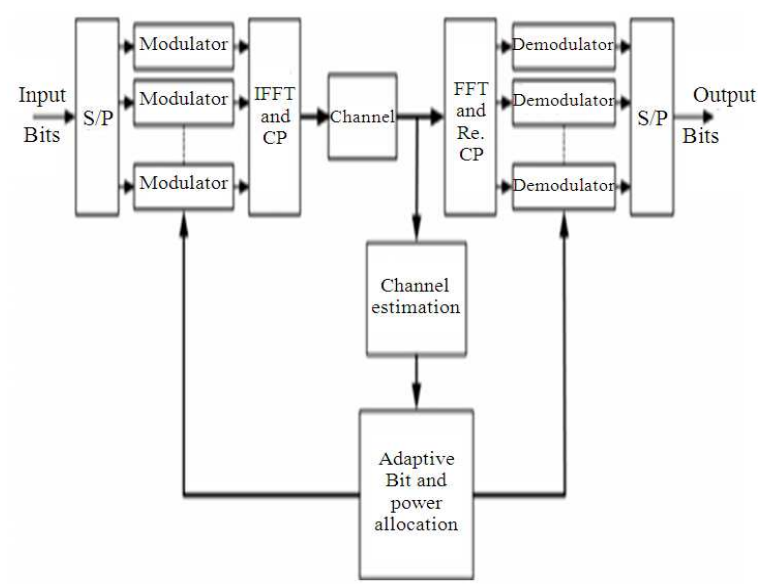

Fig. 2: OFDM system block diagram

A cyclic prefix which is set to the excess delay of the radio channel is also added to each of the resulting signals to reduce the effect of ISI and inter-sub carrier interference. The sample streams are then converted from parallel-to-serial for final transmission.

By using the OFDM technique the space time coded signals were modulated using different carrier frequencies.

\section{MATERIALS AND METHODS}

It is known that Space-Time Block Coding (STBC) has emerged as an efficient means of achieving near optimal transmitter diversity gain. And also existing implementations are sensitive to delay spreads and therefore, are limited to flat fading environments, such as indoor wireless networks. Orthogonal Frequency Division Multiplexing (OFDM) with a sufficiently long cyclic prefix can convert frequency-selective fading channels into multiple flat fading sub channels.

This study aims in providing solutions to the problems like probability of bit error rate and improving the SNR level. The present methodology is modified in order to achieve the needed efficiency. The number of antennas has been increased to obtain better performance.

Consider a wireless communication system with two antennas at the base station and two antennas at the remote. At each time slot, signals $\mathrm{S}(\mathrm{n})$ and $\mathrm{S}(\mathrm{n}+1)$ are transmitted simultaneously from the transmit antennas.

At the first time slot two bits arrive at the encoder and select two complex symbols $\mathrm{S}(\mathrm{n})$ and $\mathrm{S}(\mathrm{n}+1)$. At the first time slot two bits arrive at the encoder and select two complex symbols $S(n)$ and $S(n+1)$..After this coding each time slot signals are modulated using OFDM technique .In order to reduce the inter carrier interference each carrier signals are separated by proper cyclic prefix.

These symbols are transmitted simultaneously from antennas one and two, respectively. At the second time slot, signals $S^{*}(\mathrm{n})$ and $\mathrm{S} *(\mathrm{n}+1)$ are transmitted simultaneously from antennas one and two, respectively.

The transmission matrix can be given as:

$$
\mathrm{S}=\left(\begin{array}{cc}
\mathrm{S}(\mathrm{n}) & \mathrm{S}(\mathrm{n}+1) \\
-\mathrm{S} *(\mathrm{n}+1) & \mathrm{S} *(\mathrm{n})
\end{array}\right)
$$

The channel matrix can be given as:

$$
\mathrm{H}=\left(\begin{array}{cc}
\mathrm{h} 1 & \mathrm{~h} 2 \\
-\mathrm{h} 1 * & \mathrm{~h} 2 *
\end{array}\right)
$$

The Received signals can be obtained as:

$$
\begin{gathered}
Y(n)=h 1 s(n)+h 2 s(n+1)+v(n) \\
Y(n+1)=h 1 s *(n+1)-h 2 s *(n)+v(n+1)
\end{gathered}
$$

where, $\mathrm{v}(\mathrm{n})$ and $\mathrm{v}(\mathrm{n}+1)$ are noise components added in the channel.

The transmission model is:

$$
\mathrm{Y}=\mathrm{HS}+\mathrm{V}
$$

The matrix form of $\mathrm{V}$ is:

$$
\mathrm{V}=\left(\begin{array}{c}
\mathrm{v}(\mathrm{n}) \\
\mathrm{v} *(\mathrm{n}+1)
\end{array}\right)
$$

After coding and modulating mapping can be done for various combinations as follows.

The coding and modulations were performed for various combination of input and output antennas. After coding and modulation mapping has to be performed before the signal was fed into the transmitting antenna. Different kinds of combinations are given below.

By increasing the number of antenna at the transmitter side the capacity of the system can be increased linearly. The signal constellations for various combinations are discussed here:

$$
\begin{aligned}
& \text { Real contellation } 2 \times 2, r=1 X=\left[\begin{array}{cc}
x_{1} & x_{2} \\
-x_{2} & x_{1}
\end{array}\right] \\
& \text { Re al contellation } 4 \times 4, r=1 X=\left[\begin{array}{cccc}
x_{1} & x_{2} & x_{3} & x_{4} \\
-x_{2} & x_{1} & -x_{4} & x_{3} \\
-x_{3} & x_{4} & x_{1} & -x_{2} \\
-x_{4} & -x_{3} & x_{2} & x_{1}
\end{array}\right]
\end{aligned}
$$


Complex constellation, $\mathrm{M}_{\mathrm{t}} \times 2, \mathrm{r}=1$ :

$$
\mathrm{x}=\left[\begin{array}{cc}
\mathrm{x}_{1} & \mathrm{x}_{2} \\
-\mathrm{x}_{2}^{*} & \mathrm{x}_{1}^{*}
\end{array}\right]
$$

Complex constellation, $\mathrm{M}_{\mathrm{t}} \times 3, \mathrm{r}=3 / 4$ :

$$
\mathrm{x}=\left[\begin{array}{ccc}
\mathrm{x}_{1} & \mathrm{x}_{2} & \frac{\mathrm{x}_{3}}{\sqrt{2}} \\
-\mathrm{x}_{2}^{*} & \mathrm{x}_{1}^{*} & \frac{\mathrm{x}_{3}}{\sqrt{2}} \\
\frac{\mathrm{x}_{3}^{*}}{\sqrt{2}} & \frac{\mathrm{x}_{3}^{*}}{\sqrt{2}} & \frac{-\mathrm{x}_{1}-\mathrm{x}_{1}^{*}+\mathrm{x}_{2}-\mathrm{x}_{2}^{*}}{2} \\
\frac{-\mathrm{x}_{3}^{*}}{\sqrt{2}} & \frac{-\mathrm{x}_{3}^{*}}{\sqrt{2}} & \frac{\mathrm{x}_{2}-\mathrm{x}_{2}^{*}+\mathrm{x}_{1}-\mathrm{x}_{1}^{*}}{2}
\end{array}\right]
$$

\section{RESULTS}

MIMO system implemented using STBC with OFDM has many advantages. The BER reduces with increasing the diversity order and the SNR and BER were compared for various combinations of transmitter antennas and receive antennas. Figure 3 shows the results for basic diversity system and with OFDM system. Figure 4 shows the comparison for layered architecture for MIMO system for various number of transmit antennas and receive antennas, Fig. 5 is the output for various combination of antennas for different mapping, Fig. 6 shows the comparison of coded MIMO with uncoded MIMO.

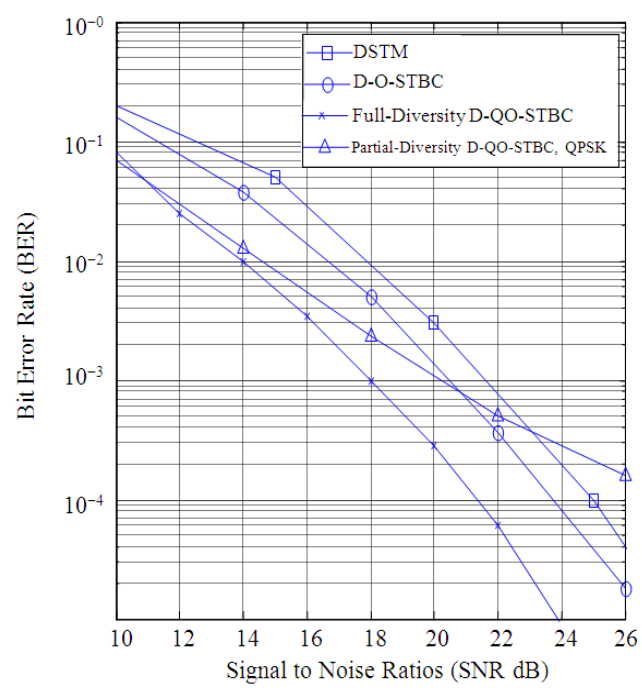

Fig. 3: Basic STBC system for various modulations

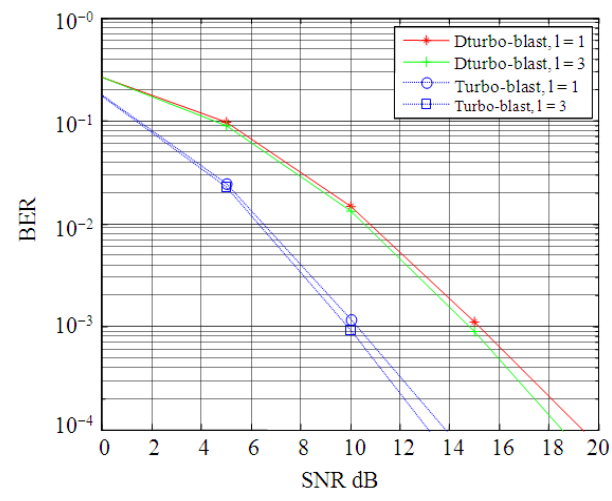

Fig. 4: STBC system with layered architecture

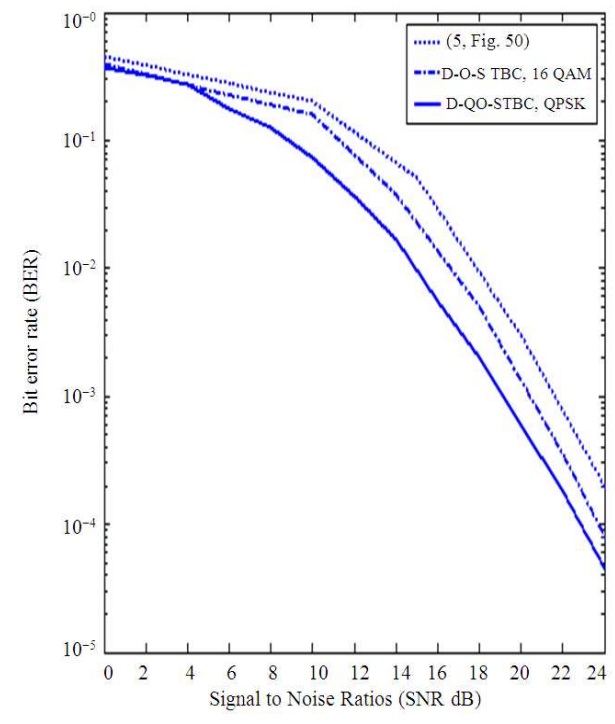

Fig. 5: STBC system implemented with OFDM

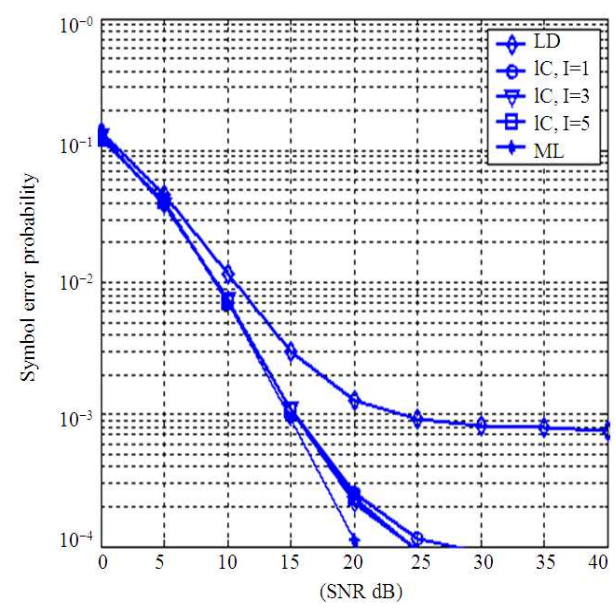

Fig. 6: STBC system implemented with OFDM 


\section{DISCUSSION}

The results show that there is an improved performance in the SNR when comparing the coded MIMO-OFDM with the un coded MIMO-OFDM system. By using different mapping technique the capacity can be increased and SNR can be improved.

Even though the complexity increases in implementing the system the connectivity increases.

\section{CONCLUTION}

Using the coded multiple antennas in both transmitter and receiver the bit error rate reduces and SNR increases. The capacity of the system also increases linearly with the increase number of antenna in both the transmitter and receiver.

\section{REFERENCES}

Alamouti, S.M., 1998. A simple transmit diversity technique for wireless communications. IEEE J. Select. Areas Commun., 16: 1451-1458. DOI: 10.1109/49.730453

Abreu, D.G.T.F. and R. Kohno, 2003. Orthogonal decoding of space-time block codes in fast fading. Proceeding of the IEEE International Symposium on Information Theory, Jun. 29-Jul. 4, IEEE Xplore Press, Japan, pp: 155-155. DOI: 10.1109/ISIT.2003.1228169

Dumard, C. and T. Zemen, 2007. Sphere decoder for a MIMO multi-user MC-CDMA uplink in timevarying channels. Proceedings of the IEEE International Conference on Communications, Jun. 24-28, IEEE Xplore Press, Glasgow, pp: 25802585. DOI: $10.1109 /$ ICC.2007.427
Hammons, Jr., A.R. and H.E. Gamal, 2000. On the theory of space-time codes for PSK modulation. IEEE Trans. Inform. Theory, 46: 524-542. DOI: $10.1109 / 18.825816$

Kaiser, T., 2005. Smart Antennas: State of the Art. 1st Edn., Hindawi Publishing Corporation, New York, USA., ISBN: 10: 9775945097 , pp: 876.

Liu, Y., M.P. Fitz and O.Y. Takeshita, 2002. A rank criterion for QAM space-time codes. IEEE Trans. Inform. Theory, 48: 3062-3079. DOI: 10.1109/TIT.2002.805074

Tarokh, V., A. Naguib, N. Seshadri and A.R. Calderbank, 1999. Space-time codes for high data rate wireless communication: performance criteria in the presence of channel estimation errors, mobility and multiple paths. IEEE Trans. Commun., 47: 199-207. DOI: 10.1109/26.752125

Tarokh, V., N. Seshadri and A.R. Calderbank, 1998. Space-time codes for high data rate wireless communication: Performance criterion and code construction. IEEE Trans. Inform. Theory, 44: 744765. DOI: $10.1109 / 18.661517$

Kwon, S., Y. Tani, H. Okubo and T. Shimomura, 2010. Fixed-Star Tracking Attitude Control of Spacecraft Using Single-Gimbal Control Moment Gyros. Am. J. Eng. Applied Sci., 3: 49-55. DOI: 10.3844/ajeassp.2010.49.55

Lan, T.S., 2010. Fuzzy Deduction Material Removal Rate Optimization for Computer Numerical Control Turning. Am. J. Applied Sci., 7: 10261031. DOI: 10.3844/ajassp.2010.1026.1031 